\section{PSICOLOGÍA IBEROAMERICANA}

\section{Psicología lberoamericana}

ISSN: 1405-0943

psicología.iberoamericana@uia.mx

Universidad Iberoamericana, Ciudad de

México

México

Moral de la Rubia, José

Validación de un formato simplificado del Inventario de Depresión de Beck (BDI-2)

Psicología Iberoamericana, vol. 21, núm. 1, enero-junio, 2013, pp. $42-52$

Universidad Iberoamericana, Ciudad de México

Distrito Federal, México

Disponible en: http://www.redalyc.org/articulo.oa?id=133929862006

- Cómo citar el artículo

Número completo

- Más información del artículo

Página de la revista en redalyc.org

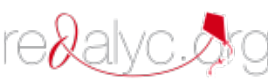

Sistema de Información Científica

Red de Revistas Científicas de América Latina, el Caribe, España y Portugal

Proyecto académico sin fines de lucro, desarrollado bajo la iniciativa de acceso abierto 


\title{
Validación de un formato simplificado del Inventario de Depresión de Beck (BDI-2)
}

\author{
Validation of a Simplified Form of Beck Depression Inventory (BDI-2)
}

José Moral de la Rubia*

Universidad Autónoma de Nuevo León

\section{RESUMEN}

Retomando un estudio previo que desarrolló con éxito una versión de aplicación simplificada del Inventario de Depresión de Beck, que hace más fácil su lectura y compresión, se hizo lo mismo con su segunda edición (BDI-2) (Beck, Steer \& Brown, 1996). Así, el objetivo de esta investigación fue estimar la consistencia interna, distribución, estructura factorial y validez concurrente de un formato simplificado del BDI-2. Se aplicaron de forma individual el BDI2, las escalas de estrés percibido de Cohen, Kamarck y Mermelstein (1983) y ansiedad-rasgo de Spielberger, Gorsuch y Lushene (1970), así como algunas preguntas sobre las creencias en las profecías del final del mundo a 424 participantes procedentes de Monterrey y con una media de 27 años. El muestreo fue no probabilístico, con cuotas equivalentes de sexos. La escala presentó una consistencia interna alta. La distribución del puntaje total del BDI-2 fue asimétrica positiva. La estructura de dos factores correlacionados (síntomas cognitivo-emocionales y somático-motivacionales) presentó un ajuste adecuado por Mínimos Cuadrados Generalizados. Las mujeres y los creyentes en las profecías del final del mundo promediaron más alto. Las correlaciones con rasgo de ansiedad y estrés percibido fueron moderadas. Se concluye que el formato ahorra espacio y tiempo, facilita la lectura y compresión, además posee tan buenas propiedades psicométricas de consistencia y validez como la escala original.

Palabras clave: BDI, depresión, neuroticismo, estrés, creencias, psicometría.

\section{ABSTRACT}

Considering a previous study that successfully developed a simplified application version of Beck Depression Inventory that makes easier its reading and compression, the same was done with the second edition (BDI-2; Beck, Steer, \& Brown, 1996). So the aims of this study were to estimate the internal consistency, distribution, factor structure and concurrent validity of a simplified form for the BDI-2. The BDI-2, the perceived stress scale (Cohen, Kamarck, \& Mermelstein, 1983) and the trait-anxiety scale (Spielberger, Gorsuch, \& Lushene, 1970), and some questions about the belief in the doomsday prophecies were applied individually to 424 participants of the general population, from Monterrey, and with an average of 27 years old. The sampling was non-probability with gender equivalent quotas. The distribution of BDI-2 total score was positively skewed. The correlated two-factor structure (cognitive-emotional symptoms and somatic-motivational symptoms) showed an adequate fit to the data by Generalized Least Squares. Women and 2012 doomsday prophecy believers averaged higher. Correlations with trait-anxiety and perceived stress were moderate. It is concluded that this application format saves space and time, facilitates reading and compression, and has as a good psychometric properties of consistency and validity as the original scale.

Keywords: BDI, depression, neuroticism, stress, psychometrics.

* Recibido: 31 de octubre, 2012 - Aceptado: 23 de marzo, 2013

e-mail: jose_moral@hotmail.com 


\section{INTRODUCCIÓN}

La depresión es un trastorno del estado de ánimo, caracterizado por tristeza o irritabilidad, abatimiento e infelicidad, y que puede ser transitorio o permanente (Beck, 2006). Belló, Puentes, Medina-Mora y Lozano (2005) reportaron que la prevalencia de depresión mayor bajo los criterios de la cuarta edición del manual diagnóstico y estadístico (DSM-IV-TR) de la Asociación Psiquiátrica Americana (APA, 2000), en adultos de población general en México en el año anterior a la aplicación de la encuesta, fue de $4.5 \%$, con $5.8 \%$ en mujeres y $2.5 \%$ en hombres.

Existen varias escalas para evaluar depresión en adultos, como la de Zung (1965), Hamilton (1967) y Goldberg, Bridges, Duncan y Grayson (1988). El Inventario de Depresión de Beck (BDI) es uno de los instrumentos de medida de sintomatología depresiva más usados, tanto en población clínica como en ámbitos de investigación con población general (García, Rivera, Reyes \& Díaz, 2011; Penley, Wiebe \& Nwosu, 2003). Desde su creación, en 1961, ha recibido revisiones menores, como el BDI-1A (Beck, Steer, Ball \& Ranieri, 1996), de las cuales la más reciente e importante es la que se publicó en 1996, de la cual surge el BDI-2 (Beck, Steer \& Brown, 1996).

El BDI se basa en las descripciones verbales que se usan con más frecuencia por los pacientes diagnosticados con depresión, ajustándose bien a la definición del trastorno depresivo mayor de la Asociación Psiquiátrica Americana (APA) en la tercera edición de su manualdiagnóstico y estadístico (DSM-III). Precisamente, el BDI-2 se creó para adaptar el inventario a los criterios de depresión mayor de la cuarta edición del DSM (Beck, 2006).

Moral (2011), desde la primera versión del BDI, desarrolla un formato simplificado de administración. En lugar de emplear una oración para cada opción de respuesta (cuatro opciones para cada una de las 21 preguntas), genera un enunciado que se responde en una escala tipo Likert de cuatro puntos, de 0 "no" a 3 "sí, mucho". Estudia las propiedades psicométricas de este nuevo formato en una muestra de participantes voluntarios conformada por 100 parejas casadas mexicanas. La escala muestra una consistencia interna alta $(\alpha=.91)$. Su distribución es asimétrica positiva ( $M=11.36$ y $D E=9.95)$, y la mediana de las mujeres
(11) es significativamente mayor que la de los hombres (8). Con base en el criterio de Cattell se definen dos factores que explicaron el $39.47 \%$ de la varianza total. El primero, de 11 ítems, corresponde a síntomas cognitivo-emocionales de depresión $(\alpha=.87)$; el segundo, de 10 a síntomas somático-motivacionales $(\alpha=.85)$. Por Mínimos Cuadrados Generalizados los índices de ajuste son adecuados ( $\chi 2 / g l=1.86, R M S E A=.07$, $G F I=.83, A G F=.80)$. Al reducir a cinco indicadores por factor el modelo factorial presenta mejor ajuste $(\chi 2 /$ $g l=2.22, R M S E A=.06, G F I=.92$ y $A G F I=.88$ ). Además se obtienen evidencias de validez, con correlaciones directas y moderadas con afecto negativo medido por la Escala de Afecto Positivo y Negativo (PANAS) de Watson, Clark y Tellegen (1988) $\left(r_{S}=.56\right)$, severidad de síntomas somáticos con la escala creada por Woolfolk y Allen (2007) $\left(r_{s}=.43\right)$ y alexitimia con la escala de Bagby, Parker y Taylor (1994) $\left(r_{s}=.41\right)$, e inversa y baja con afecto positivo medido por el PANAS $\left(r_{s}=-.18\right)$.

Debe señalarse que los 21 ítems del BDI suelen presentar, en diversos estudios, indicios claros de unidimensionalidad, ya que las correlaciones entre los dos factores son altas; no obstante, el ajuste del modelo de dos factores correlacionados es superior al unidimensional, además de ofrecer mayor valor heurístico (Beck, Steer \& Brown, 1996; Beck, 2006; Moral, 2011).

Del estudio de Moral (2011) se desprende que las propiedades psicométricas de consistencia interna y validez de la escala original pueden mantenerse equivalentes con el formato simplificado de aplicación que no sólo ahorra espacio y tiempo, sino que resulta más sencillo de leer y comprender. Este ahorro tiene en sí gran valor en investigaciones realizadas en población general en las que se aplican varios instrumentos, pero es más importante hacer una lectura y comprensión más fáciles cuando se evalúa a una población con problemas de atención y fatiga, como es la de pacientes deprimidos (Shenal, Harrison \& Demaree, 2003).

El estudio de la versión original del BDI se justificó por su amplio uso en atención primaria, aún hoy en día (Myers \& Winters, 2002; Ruscio \& Ruscio, 2002). No obstante, considerando que existe una versión más reciente (Beck, Steer \& Brown, 1996), cuyo uso está más extendido, se consideró relevante desarrollar un formato simplificado de la misma. 
Por la afinidad como afectos negativos y relación temporal de la depresión con la ansiedad, tanto estado como rasgo (Watson, 2000), el BDI ha sido con frecuencia validado en relación con escalas que miden estos constructos (Beck, 2006), siendo las correlaciones moderadas, de .40 a .60 (Hammen, 2005).

Se define rasgo de ansiedad o neuroticismo a la tendencia persistente a sobrerreacionar ante estímulos novedosos o amenazantes y mostrar gran sensibilidad al castigo y el daño; por el contario, si esta sobrerreactividad y sensibilidad son transitorias, usualmente por sobrecargas de estresores, se habla de estrés o ansiedad estado (Boksema, Topsa, Westera, Meijmana \& Lorist, 2006). Uliaszek et al. (2010) observaron a través de correlaciones parciales que el neuroticismo es una variable que media en la correlación entre la ansiedad estado o estrés, y la depresión, además de ser un antecedente importante de ambos estados de afecto negativo. Precisamente, las personas con rasgos de neuroticismo tienden a sufrir más estrés y depresión a comparación de aquellas sin este rasgo. Además, ambos estados afectivos tienden a solaparse más (Malouff, Thorsteinsson \& Schutte, 2005). Así, la correlación de la depresión es más alta con el rasgo de ansiedad que con el estrés, en un rango moderado ambas; además, la correlación entre depresión y estrés se debilita si se parcializa el efecto del neuroticismo (Uliaszek et al., 2010).

El BDI nunca se ha estudiado en relación con las creencias apocalípticas, esto es, creer que el mundo pronto se acabará, cuando se puede esperar una asociación directa (Moral, 2012) y servir así como una prueba adicional de validez convergente. Una posible explicación de la creencia en las profecías del fin de mundo como las del 2012, sería la tendencia al pensamiento mágico y la adhesión a ciertos movimientos religiosos con postulados apocalípticos (Lawrence \& Peters, 2004). No obstante, también se podría considerar el estado de ánimo. Las personas que sufren ansiedad o están deprimidas pueden albergar deseos de que todo acabe para aliviar su sufrimiento y el pesar de la vida, especialmente con un hecho que afecte a toda la humanidad y que no implique la responsabilidad de un pensamiento suicida. Así, la mayor convicción en las creencias apocalípticas podría tener su origen en un estado de ánimo negativo, concretamente de ansiedad y depresión (Moral \& Tovar, 2012).
Según lo que se expuso y se argumentó anteriormente, esta investigación tiene como objetivos: desarrollar un formato tipo Likert de respuesta para el BDI-2; contrastar una estructura de dos factores correlacionados frente a la de un factor general; describir la distribución y estimar la consistencia interna del puntaje total y los factores; comparar diferencias de medias entre ambos sexos en el puntaje total y los factores; asimismo, estudiar la relación de los mismos con neuroticismo, estrés y creencias en las profecías apocalípticas del año 2012 para aportar pruebas de validez concurrentes.

Se espera un ajuste adecuado a los datos del modelo de dos factores correlacionados (síntomas cognitivoafectivos y somático-motivacionales) y superior al modelo unidimensional, valores de consistencia interna altos, distribuciones asimétricas positivas, correlaciones moderadas con rasgo de ansiedad y estrés, mayores medias en mujeres y en los que creen en las profecías del final del mundo

\section{MÉTODO}

La presente investigación es un estudio instrumental, descriptivo-correlacional con un diseño no experimental transversal.

\section{Participantes}

Se realizó un muestreo no probabilístico. Se recolectó una muestra con cuotas equivalentes de sexos, de 424 participantes (212 mujeres y 212 hombres) de población general, provenientes del área metropolitana del estado de Nuevo León, con un rango de edad de 18 a 65 años, media de 27.09 y desviación estándar de 11.25. El 3\% (11 de 424) reportó tener estudios de primaria, 9\% (39) de secundaria, 22\% (95) media-superior y $66 \%$ (279) universitarios. El 76\% (324 de 424) dijo ser católico, 7.5\% (32) cristiano no católico, 2\% (7) otra religión, $10 \%$ (42) ninguna en particular y $4.5 \%$ (19) agnóstico o ateo.

\section{Instrumentos}

Escala de estrés percibido (Percieved Stress Scale [PSS14]; Cohen, Kamarck \& Mermelstein, 1983). Se emplea la adaptación a población mexicana de González y Landero (2007). La escala evalúa el grado en que los participantes valoran las situaciones del último mes como 
impredecibles y fuera de control. Consta de 14 ítems tipo Likert con un rango de cinco puntos, de 0 "nunca" a 4 "siempre". Para obtener la puntuación total de PSS se deben invertir las puntuaciones de los ítems: 4, 5, $6,7,9,10$ y 13 (restando a 4 el valor del ítem) y sumar los 14 ítems. El rango varía entre 0 (mínimo estrés) y 56 (máximo). El valor del coeficiente alfa para los 14 ítems en muestras estadounidenses varió de .84 a .86 (Cohen et al., 1983) y fue de .83 entre estudiantes universitarios mexicanos (González \& Landero, 2007). La escala consta de dos factores correlacionados: control del estrés con los siete ítems inversos y estrés descontrolado con los siete ítems directos.

Escala de rasgo del Inventario de Ansiedad (State Trait Anxiety Inventory- Trait scale [TA], Spielberger, Gorsuch \& Lushene, 1970). Se emplea la traducción realizada para población mexicana por Spielberger y Díaz-Guerrero (1975). Se usaron sólo los dos últimos factores: el de afirmación de rasgo de ansiedad, que está integrado por 13 ítems $(2,3,4,5,8,9,11,12,14$, $15,17,18$ y 20) y el de negación del rasgo de ansiedad, que está compuesto por siete ítems $(1,6,7,10,13,16$ y 19). El formato de respuesta es tipo Likert con cuatro puntos de rango, de 0 "nunca" a 3 "casi siempre". La consistencia interna de estos dos factores varía de .85 a .87 (Spielberger \& Díaz-Guerrero, 1975).

Inventario de Depresión de Beck, segunda edición (BDI-2; Beck, Steer \& Brown, 1996) con un formato de aplicación simplificado. El BDI-2 consta de 21 ítems tipo Likert con un rango de respuestas de 0 "nada" a 3 "mucho". Todos son directos, lo que hace posible reflejar una mayor puntuación más sintomatología depresiva.

También se incluye una pregunta sobre las profecías del fin del mundo: ¿piensa que próximamente se cumplirán las profecías del final del mundo o de una etapa evolutiva de la humanidad?, con opciones de respuesta: "sí, en el final del mundo", "sí, en el final de una etapa evolutiva" y "no". Esta pregunta es antecedida por una escala de pensamiento mágico creada por Moral (2009) que no se considera en estos análisis. De ahí seguía el PSS-14, TAI y BDI-2. La misma secuencia se aplicó a todos los participantes para evitar distorsiones generadas por la activación de esquemas ansiógenos o depresógenos (Beck, 2006).

\section{Procedimiento}

Se realizó un estudio descriptivo-correlacional con un diseño ex posfacto transversal. Los encuestadores fueron estudiantes de psicología que cursaban una asignatura de investigación, en la cual se requería colaborar en un trabajo de campo como parte de los créditos. Se pidió a cada alumno aplicar el cuestionario a ocho mujeres y ocho hombres adultos, independientes entre sí, dejando que el participante contestase por sí mismo (autoinforme).

El cuestionario era respondido en presencia del encuestador, apoyando al participante en caso de dudas; o bien era entregado y recogido posteriormente, comprobando que se hubiera respondido a todos los ítems. En caso de omisiones, se solicitaba la respuesta y se prestaba apoyo para comprender la pregunta si el encuestado así lo requería. De forma previa se dio capacitación para tal fin. El trabajo de campo se realizó de abril a julio de 2011. Se anularon ocho cuestionarios por respuestas incompletas.

Una hoja de consentimiento manifiesto informado encabezaba el cuestionario. En caso de no querer participar se pedía indicar el porqué dentro de una lista de opciones. Se consideró que, cuando algún participante solicitase atención psicológica, fuera canalizado a los servicios de la Facultad de Psicología con asistencia gratuita, lo que no ocurrió en ningún caso. Se respetó la confidencialidad de la información en el manejo de los datos. Así, el estudio se ajustó a las normas éticas de investigación de la American Psychological Association (2002) y la Sociedad Mexicana de Psicología (2007).

\section{Análisis de datos}

Se calculó la consistencia interna por el coeficiente alfa de Cronbach. Se contrastó el ajuste de la distribución a una curva normal con la prueba de KolmogorovSmirnov, empleando la corrección de Lilliefors en el cálculo de la probabilidad para obtener más potencia estadística.

La estructura factorial se estudió con análisis factorial exploratorio y confirmatorio, desde las hipótesis de una solución uni o bidimensional. Para el exploratorio se empleó el análisis de componentes principales con rotación Oblimín. A su vez, se contrastaron ambos modelos por Análisis Factorial Confirmatorio (AFC). 
Los parámetros e índices de ajuste se estimaron por Mínimos Cuadrados Generalizados (GLS). Se manejaron siete índices de ajuste: función de discrepancia $(F D)$, estadístico chi-cuadrado $(\chi 2)$, cociente entre el estadístico chi-cuadrado y sus grados de libertad $\left(\chi^{2 /}\right.$ $g l)$, parámetro de no centralidad poblacional (PNCP), residuo cuadrático medio de aproximación (RMSEA) de Steiger-Lind, índice de bondad de ajuste (GFI) de Jöreskog y Sörbom y su modalidad corregida (AGFI). Se estipularon como valores de buen ajuste: $p$ de $\chi 2>$ $.05, F D$ y $\chi 2 / g l<2, P N C P<1, R M S E A \leq .05, G F I \geq .95$ y $A G F I \geq .90$; y los valores adecuados: $p$ de $\chi 2>.01$, $F D$ y $\chi 2 / g l \leq 3, P N C P \leq 2, R M S E A<.08, G F I \geq .85$ y $A G F I \geq .80$ (Moral, 2006). Para comparar el ajuste de los modelos de un factor general y de dos factores correlacionados se empleó la prueba de la diferencia de los estadísticos chi-cuadrado $(\Delta \chi 2)$ (Yuan \& Bentler, 2004).

Las correlaciones con estrés y rasgo de ansiedad se calcularon con el coeficiente producto-momento de Pearson $(r)$. Las diferencias de medias por sexos se contrastaron por la prueba $t$ de Student y entre los tres grupos de creencias en las profecías de 2012, por el análisis de varianza.

Los cálculos estadísticos se realizaron con SPSS versión 16 y AMOS versión 16.

\section{RESULTADOS}

\section{Estructura factorial}

Los modelos de un factor general y dos factores correlacionados, en la matriz de correlaciones de este estudio, se estudian primero a nivel exploratorio por componentes principales y a continuación a nivel confirmatorio, por mínimos cuadrados generalizados (GLS).

Por el criterio de Cattell se podría definir un único componente, al ubicarse el punto de inflexión de la curva de sedimentación en el segundo autovalor (figura 1). Con un componente se explica el $38.16 \%$ de la varianza total, las cargas factoriales varían de .74 a .37 y la consistencia interna es alta $(\alpha=.91)$.

Hay un segundo punto de inflexión en el tercer autovalor (figura 1), por lo que también se podría definir una solución bidimensional. Estos dos componentes explican el $47.35 \%$ de la varianza total. Se obtiene un primer componente de síntomas cognitivo-afectivos (del 1 al 14) con consistencia interna alta $(\alpha=.90)$, y otro segundo de síntomas somático-motivacionales (del 15 al 21) también con consistencia interna alta $(\alpha=.83)$. La correlación entre ambos componentes es significativa, directa y moderada $(r=.48, p<.01)$.

Por análisis factorial confirmatorio por el método GLS, se contrasta un modelo unidimensional [1F] y el de dos factores correlacionados [2F-Cor.] (figura 2). El ajuste es diferencialmente mejor para el modelo bidimensional: $\Delta \chi^{2}(1, N=424)=51.42, p<.01$. Los índices de ajuste del modelo de dos factores correlacionados son adecuados: $\chi 2 / g l=2.80, G F I=.88$, $A G F I=.85, F D=1.24, P N C P=0.80$ y $R M S E A=.06$. Todos los parámetros resultan significativos, fluctuando la varianza explicada de los ítems de 20 al 61\%, con una media de $45 \%$. La correlación entre ambos factores es alta $(r=.77, p<.01)$, compartiendo el $59 \%$ de la varianza, de ahí que son claramente discernibles.

\section{Figura 1. Curva de sedimentación de los 21 autovalores}

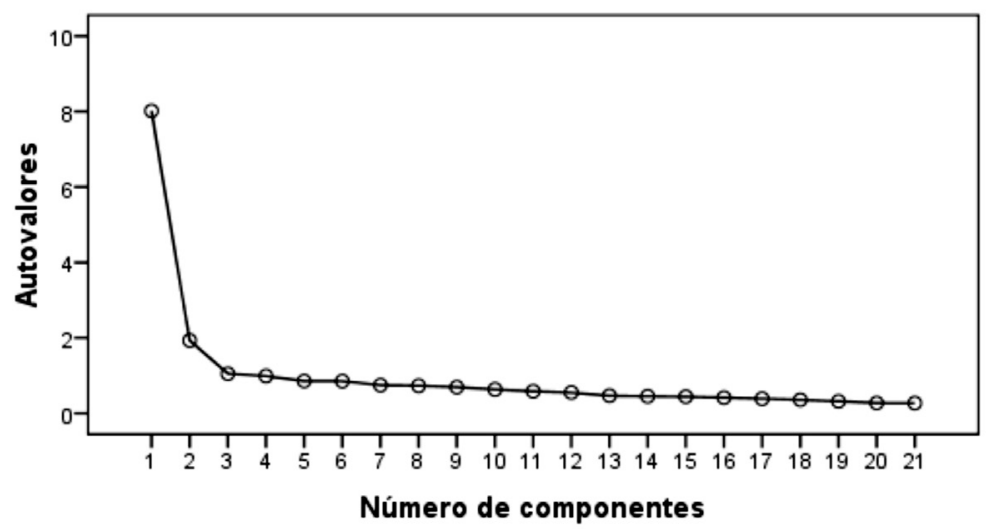


Figura 2. Modelo estandarizado de dos factores correlacionados (estimado por GLS) para los 21 ítems del BDI-2 (modelo 2F-Cor)

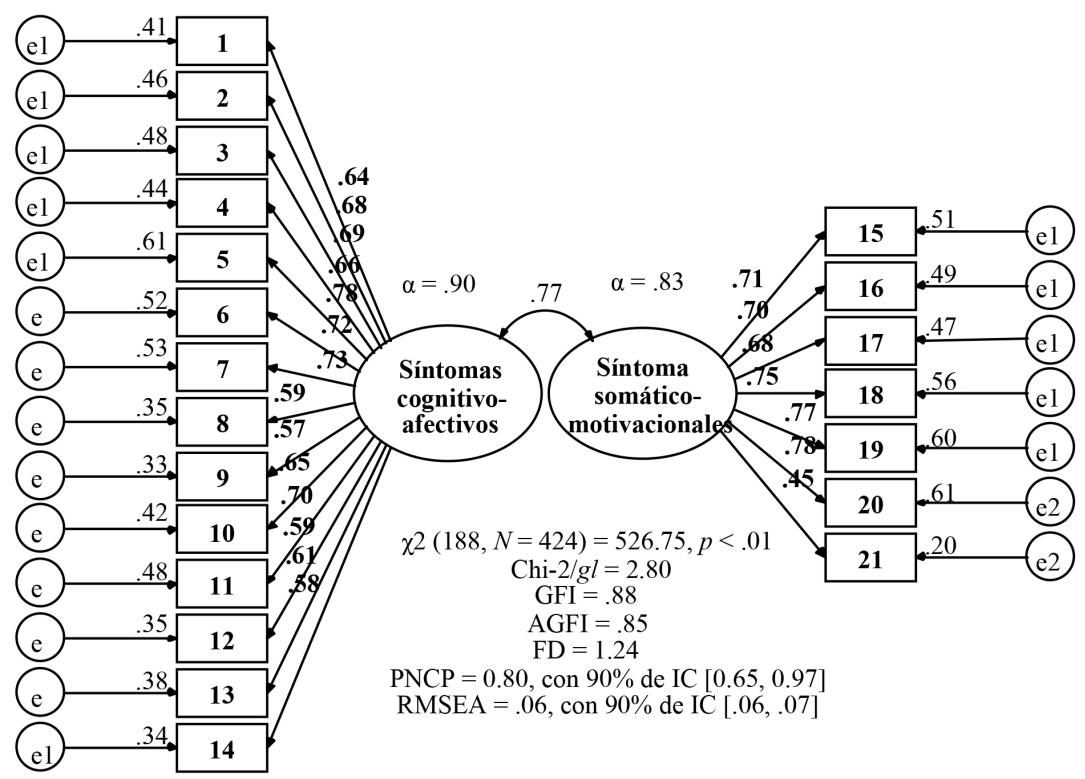

\section{Descripción de las distribuciones}

La distribución del puntaje total del BDI-2 de media $11.58(D E=9.66)$ es asimétrica positiva y apuntada, no se ajusta a una curva normal por la prueba de
Kolmogorov-Smirnov $\left(Z_{K-S}=2.56, p<.01\right)$, al igual que sus factores de síntomas cognitivo-afectivos y síntomas somático-motivacionales.

Tabla 1. Consistencia interna, descriptivos y ajuste a la normalidad del puntaje total y los dos factores de BDI-2

\begin{tabular}{|c|c|c|c|c|}
\hline \multicolumn{2}{|c|}{ Estadísticos } & \multirow{2}{*}{$\frac{\text { Puntaje total }}{21}$} & \multirow{2}{*}{$\frac{\text { Cognitivo-afectivos }}{14}$} & \multirow{2}{*}{$\begin{array}{c}\text { Somático-motivacionales } \\
7\end{array}$} \\
\hline Concistonci & No de ítems & & & \\
\hline comsistemcia & & .91 & .90 & .83 \\
\hline \multirow{14}{*}{ Descriptivos } & Rango & $0-21$ & $0-42$ & $0-21$ \\
\hline & $M$ & 11.58 & 5.95 & 5.64 \\
\hline & $\mathrm{DE}$ & 9.66 & 6.44 & 4.34 \\
\hline & $S$ & 1.16 & 1.68 & 0.73 \\
\hline & C & 1.37 & 3.05 & 0.04 \\
\hline & P10 & 1 & 0 & 0 \\
\hline & P20 & 3 & 1 & 2 \\
\hline & P30 & 5 & 2 & 3 \\
\hline & P40 & 7 & 3 & 4 \\
\hline & P50 & 9 & 4 & 5 \\
\hline & P60 & 12 & 5 & 6 \\
\hline & P70 & 15 & 7 & 8 \\
\hline & P80 & 20 & 10 & 9 \\
\hline & P90 & 25 & 14.5 & 12 \\
\hline \multirow{3}{*}{$\begin{array}{l}\text { Ajuste a la } \\
\text { normalidad }\end{array}$} & $\mathrm{D}$ & .12 & .18 & .12 \\
\hline & ZK-S & 2.56 & 3.67 & 2.49 \\
\hline & $p^{*}$ & $>.01$ & $>.01$ & $>.01$ \\
\hline
\end{tabular}

$S=$ Sesgo, $E E$ de $S=.12 . C=$ Curtosis, $E E$ de $C=.24 . p^{*}$ con la corrección de Lilliefors para distribución normal. 


\section{Comparación entre los sexos}

Por la prueba $t$ de Student las mujeres promedian significativamente más alto que los hombres en el puntaje total y síntomas cognitivo-afectivos; sin embargo, las medias son estadísticamente equivalentes entre ambos sexos en síntomas somático-motivacionales.

\section{Validez concurrente con ansiedad y creencias apocalípticas}

Las correlaciones del puntaje total y los dos factores del BDI-2 son significativas con el puntaje total y los dos factores del PSS-14 y del TA, variando de .67 a -.20.

El 7.6\% (32 de 424) de los participantes manifestó creer en las profecías de final del mundo en el 2012; $37 \%$ (157 de 424) en que se terminará una etapa evolutiva de la humanidad y $55.4 \%$ (235 de 424 ) no cree en dichas profecías. Las diferencias de medias en el puntaje total y los dos factores del BDI-2 son significativas. Se contrastan las diferencias por pares por la prueba de Tamahane (sin asumir igual de varianza) con el puntaje total y el factor de síntomas cognitivo-afectivos y por la prueba de la Diferencia Mínima Significativa (asumiendo igual de varianza) con el factor de síntomas somático-motivacionales.

Los que creen en el final del mundo promedian significativamente más alto en el puntaje total y los dos factores en comparación con los que no creen en las profecías, y en el puntaje total y el factor de síntomas cognitivo-afectivos, en comparación con los que creen en el final de una etapa evolutiva. Entre los no creyentes y los que creen en el final de una etapa evolutiva los promedios son estadísticamente equivalentes.

Tabla 2. Diferencia de medias en el BDI-2 por sexos

\begin{tabular}{|c|c|c|c|c|c|c|c|c|c|}
\hline \multirow{2}{*}{ BDI-2 } & \multicolumn{2}{|c|}{ Mujeres } & \multicolumn{2}{|c|}{ Hombres } & \multicolumn{2}{|c|}{ Diferencia } & \multicolumn{3}{|c|}{ Contraste } \\
\hline & $M$ & $D E$ & $M$ & $D E$ & $M$ & $E E$ & $t$ & $g l$ & $p$ \\
\hline Puntaje total & 12.60 & 10.29 & 10.57 & 8.90 & 2.04 & 0.93 & $2.18^{*}$ & 422 & .03 \\
\hline S. cognitivo-afectivos & 6.60 & 7.00 & 5.29 & 5.77 & 1.32 & 0.62 & $2.11 * *$ & 407.19 & .03 \\
\hline S. somático-motivacionales & 6.00 & 4.52 & 5.28 & 4.14 & 0.72 & 0.42 & $1.71 *$ & 422 & .09 \\
\hline
\end{tabular}

Sexo: $M=$ Mujer $(n=212)$ y $\mathrm{H}=$ Hombre $(n=212)$.

Asumiendo igualdad de varianza con base en la prueba de Levene.

** Sin asumir igualdad de varianza.

Tabla 3.Correlaciones del BDI-2 con TA y PSS-14

\begin{tabular}{l|l|c|c|c}
\hline \multirow{2}{*}{ Ansiedad } & \multicolumn{2}{|c}{ Depresión (BDI-2) } \\
\cline { 3 - 5 } & Puntaje total de estrés & Puntaje total & Cognitivo-afectivos & Somático-motivacionales \\
\hline \multirow{3}{*}{ PSS-14 } & Control del estrés & $.51^{* *}$ & $.50^{* *}$ & $.39^{* *}$ \\
\cline { 2 - 5 } & Estrés descontrolado & $-.31^{* *}$ & $-.34^{* *}$ & $-.20^{* *}$ \\
\hline \multirow{2}{*}{ TA } & Puntaje total & $.39^{* *}$ & $.35^{* *}$ & $.34^{* *}$ \\
\cline { 2 - 5 } & Afirmación de rasgos de ansiedad & $.64^{* *}$ & $.64^{* *}$ & $.54^{* *}$ \\
\cline { 2 - 5 } & Negación de rasgos de ansiedad & $-.38^{* *}$ & $.59^{* *}$ & $-.26^{* *}$ \\
\hline
\end{tabular}

$p<.01 . N=424$. 


\section{DISCUSIÓN}

Se confirma la expectativa de dos factores correlacionados con la misma interpretación y composición que la que se ha reportado en otros estudios, como el original de Beck, Steer y Brown (1996), con un ajuste diferencialmente superior al modelo unidimensional. También se confirman los valores de consistencia interna altos para el conjunto de 21 ítems y dos factores. Esta consistencia alta del conjunto de ítems justifica claramente el uso del puntaje total.

Según la expectativa, la distribución del puntaje total se sesga hacia los valores bajos y es apuntada, lo que refleja ausencia de depresión en la mayoría de los participantes, especialmente al tratarse de una muestra de población general. Al ser la característica evaluada un estado patológico, la distribución se aleja del modelo matemático de una curva normal, por lo que requiere ser estandarizada por los percentiles en lugar de la media y desviación estándar (Sartori, 2006).

Beck et al. (1996) establecieron que puntuaciones de 0 a 13 indican ausencia de depresión o síntomas mínimos; de 14 a 19, depresión leve; de 20 a 28, moderada; y de 29 a 63, severa. Debe señalarse que el punto de corte de 14 (Beck, Steer \& Brown, 1996) sería inadecuado para definir depresión leve, pues corresponde al percentil 65. La puntuación de 15, que concierne al percentil 70, sería más adecuada para clasificar como posible caso de depresión. Si se considera que la prevalencia de la depresión en población general de adultos estadounidenses varía del 10 al 25\% para las mujeres y del 5 al 12\% para los varones (American Psychiatric Association [APA], 2000), se podría estipular un porcentaje de $10 \%$ de posibles casos de depresión en la muestra; así se requeriría una puntuación de 25 o mayor desde el percentil 90. No obstante, la estimación de prevalencia de depresión mayor de Belló et al. (2005) en México es más baja y habría que acudir al percentil 95 con una puntuación de 29. Esto se asemeja a lo que Sanz, García, Espinosa, Fortún y Vázquez (2005) propusieron en España con una puntuación mayor o igual a 15 para población clínica en estudios de cribado (posible caso), e igual o mayor a 30 para diagnóstico clínico (caso).

Hay que destacar que la media de 11.58 ( $D E=9.66)$ del puntaje total del BDI-2 de la presente muestra neoleonesa de población general es significativamente equivalente $(D M=0.22, t(423)=0.48, p=.63)$ a la media de $11.36(D E=9.95)$ de otra muestra de 200 parejas casadas neoleonesas, donde se aplicó el BDI-1 con el mismo formato simplificado de respuesta (Moral, 2011). Asimismo, en una muestra de 178 estudiantes universitarios estadounidenses, Wiebe y Penley (2005) obtuvieron una media de $11.50(D E=9.2)$ con la versión en inglés del BDI-2 en su formato de aplicación original y de $11.70(D E=7.4)$ con la versión en español del BDI-2 en una muestra de 76 estudiantes hispanos. En ambos casos las medias son equivalentes con la presente $(D M=0,08, t[423]=0.18, p=.85$ para la versión en inglés y $D M=-0,11, t[423]=-0.24, p=.81$ para la versión en español). Por lo tanto, el punto de corte de 14 propuesto por Beck, Steer y Brown (1996) parece bajo. La diferencia entre los sexos en las escalas de depresión, incluyendo el BDI, es un hecho bien establecido (Beck, 2006; Keogh, McCracken \& Eccleston, 2006), tal como también se observa en los estudios epidemiológicos con entrevistas estructuradas (APA, 2000; Belló et al., 2005). En la presente investigación se constata dicha diferencia, ya que se observa mayor media en mujeres, lo que constituye una prueba de validez. Las mujeres, especialmente en edades fértiles, son más susceptibles a los trastornos afectivos tanto por determinantes biológicos en relación con el ciclo menstrual, que afecta a la disponibilidad de la serotonina cerebral (Toufexis, Myers \& Davis, 2006), como por determinantes culturales que fomentan el afrontamiento pasivo y la dependencia interpersonal (Piccinelli \& Wilkinson, 2000; Rocha \& Díaz-Loving, 2005).

Hay evidencia de validez en relación con los criterios convergentes de rasgo de ansiedad y estrés. La asociación es moderada, al ser constructos claramente discernibles (Watson, 2000). El BDI-2 comparte más varianza con el rasgo de ansiedad que con el estado de estrés percibido, como se esperaba. El rasgo implica una mayor carga de afectividad negativa crónica que conlleva más vivencias depresivas que el estado de estrés (McCrae \& Costa, 2004), y es un factor de riesgo tanto de estrés como depresión (Uliaszek et al., 2010). Precisamente, al parcializar el efecto de la ansiedad, rasgo en la correlación entre la depresión y el estrés, ésta pasa de ser moderada $(r=.51)$ a ser baja $\left(r_{p}=.13\right)$. Aparte se podría considerar la mayor amplitud y afinidad de los contenidos del TA al BDI-2 en compara- 
ción con el PSS-14. Así, Caci, Baylé, Dossios, Robert y Boyer (2003) remarcan que el TA incluye al menos seis ítems con claro contenido depresivo.

Dentro del BDI-2 las correlaciones son más altas con el factor de síntomas cognitivo-afectivos que somático-motivacionales, probablemente por la mayor especificidad para depresión de los mismos. La escala PSS-14 no hace referencia a síntomas somáticos; por el contario, evalúa estrés percibido que es la dimensión cognitiva de la ansiedad; de ahí que las correlaciones más bajas sean entre PSS-14 y el factor de síntomas somático-motivacionales del BDI-2.

En la presente muestra domina el no creer en las profecías apocalípticas para el año 2012. Más de la mitad no creen en las profecías, superando en una décima parte a los que sí lo hacen. Además, entre quienes creen, es más frecuente hablar del final de una etapa evolutiva que de final del mundo. Se argumentó que el pesimismo, desgana de vivir y deseo de muerte que caracteriza la depresión motivarían un apoyo a la creencia en el final del mundo bajo un deseo de que acabe el sufrimiento mental sin la responsabilidad del suicidio (Moral \& Tovar, 2012). La asociación se confirma en este estudio, lo que aporta validez al formato creado para el BDI-2; no obstante, la confirmación del mecanismo hipotetizado requeriría estudios adicionales de corte cualitativo para profundizar en la vivencia de fondo.

Dadas las características no probabilísticas de la muestra empleada, los resultados deben ser manejados como hipótesis para futuras investigaciones y no como estimaciones poblacionales. No obstante, el tamaño de la misma proporciona potencia en las pruebas de contraste (Murphy \& Myors, 2003). Además, debe considerarse la naturaleza de autorreporte de los datos, ya que éstos pueden diferir de los obtenidos por otros medios, como entrevistas o técnicas proyectivas.
En conclusión, se confirma la estructura de dos factores correlacionados (síntomas cognitivo-afectivos y somático-motivacionales) con un ajuste diferencialmente mejor al modelo unidimensional, valores de consistencia interna altos, distribuciones asimétricas positivas y apuntadas que requieren ser baremadas por percentiles, diferencias de sexos con mayor promedio en mujeres, correlaciones moderadas con rasgo de ansiedad y estrés percibido, siendo mayor la correlación con rasgo que con estrés, asimismo, promedios más altos en personas que creen en el final del mundo para el año 2012 en comparación con los que creen en el final de una etapa evolutiva y los no creyentes en tales profecías. Los resultados de estructura factorial, consistencia interna y tipo de distribución son equivalentes al formato original de la escala publicado en 1996 y validado en diversos países (Melipillán, Cova, Rincón \& Valdivia, 2008; Sanz, Perdigón \& Vázquez, 2003), por lo que este formato de aplicación que facilita la lectura y compresión, además de ahorrar tiempo y espacio, es confiable y válido, por lo que se recomienda su uso.

El punto de corte de 14 es bajo para este formato de aplicación. Se propone una puntuación de 15 o mayor para clasificar como posible caso de depresión desde el percentil 70 de la presente muestra, y una puntuación de 29 o mayor para clasificar como caso de depresión mayor desde el percentil 95, al considerar una prevalencia del 5\% en población mexicana con base en el estudio de Belló et al. (2005).

Se sugiere, para futuros estudios, establecer los estándares de la escala por medio de dos muestras probabilísticas de población clínica y abierta; asimismo, confirmar los resultados de este investigación hecha en una muestra no probabilística de población abierta. • 


\section{REFERENCIAS}

American Psychiatric Association (2000). Diagnostic and statistical manual of mental disorders (4a. Ed., text revision). Washington, DC: American Psychiatric Association.

American Psychological Association (2002). Ethical principles of psychologists and code of conduct. American Psychologist, 57, 1060-1073.

Bagby, R. M., Parker, J. D. A. \& Taylor, G. J. (1994). The twenty-item Toronto alexithymia scale-I. Item selection and cross-validation of the factor structure. Journal of Psychosomatic Research, 38, 23-32.

Beck, A. T. (2006). Depression: Causes and treatment. Filadelfia: University of Pennsylvania Press.

Beck, A. T., Steer, R. A., Ball, R. \& Ranieri, W. (1996). Comparison of Beck Depression Inventories -IA and -II in psychiatric outpatients. Journal of Personality Assessment, 67, 588-597.

Beck, A. T., Steer, R. A. \& Brown, G. K. (1996). Manual for the Beck Depression Inventory-II. San Antonio, TX: Psychological Corporation.

Belló, M., Puentes, E., Medina-Mora, M. E. \& Lozano, R. (2005). Prevalencia y diagnóstico de depresión en población adulta en México. Salud Pública de México, 47(supl. 1), 4-11.

Boksema, M. A. S, Topsa, M., Westera, A. E., Meijmana, T. F. \& Lorist, M. M. (2006). Error-related ERP components and individual differences in punishment and reward sensitivity. Brain Research, 1101(1), 92-101.

Caci, H., Baylé, F. J., Dossios, C., Robert, P. \& Boyer, P. (2003). The Spielberger Trait Anxiety Inventory measures more than anxiety. European Psychiatry, 18, 394-400.

Cohen, S., Kamarck, T. \& Mermelstein, R. (1983). A global measure of perceived stress. Journal of Health and Social Behavior, 24, 385-396.

García, M., Rivera, S., Reyes, I. \& Díaz, R. (2011). El enfrentamiento y el conflicto: factores que intervienen en la depresión. Acta de Investigación Psicológica, 1, 415-427.

Goldberg, D., Bridges, K., Duncan-Jones, P. \& Grayson, D. (1988). Detecting anxiety and depression in general medical settings. British Medical Journal, 297, 897-899.
González, M. T. \& Landero, R. (2007). Factor Structure of the Perceived Stress Scale (PSS) in a Sample from Mexico. Spanish Journal of Psychology, 10, 199-206.

Hamilton, M. (1967). Development of a rating scale for primary depressive illness. British Journal of Social and Clinical Psychology, 6, 278-296.

Hammen, C. (2005). Stress and depression. Annual Review of Clinical Psychology, 1, 293-319.

Keogh, E., McCracken, L. M. \& Eccleston, C. (2006). Gender moderates the association between depression and disability in chronic pain patients. European Journal of Pain, 10, 413-422.

Lawrence, E. \& Peters, E. (2004). Reasoning in believers in the paranormal. Journal of Nervous y Mental Disease, 192, 727-733.

Malouff, J. M., Thorsteinsson, E. B. \& Schutte, N. S. (2005). The relationship between the five factor model of personality and symptoms of clinical disorders. Journal of Psychopathology and Behavioral Assessment, 27(2), 101-114.

McCrae, R. R. \& Costa, P. T. (2004). A contemplated revision of the NEO five-factor inventory. Personality and Individual Differences, 36, 587-596.

Melipillán, R. Cova, F., Rincón, P. \& Valdivia, M. (2008). Propiedades psicométricas del Inventario de Depresión de Beck-II en adolescentes chilenos. Terapia Psicológica, 26(1), 59-69.

Moral, J. (2006). Análisis factorial confirmatorio. En R. Landero \& M. T. González (Eds.), Estadística con SPSS y metodología de la investigación (pp. 445-528). México: Trillas.

Moral, J. (2009). Escala para medir superstición y pensamiento mágico. Bogotá: Psicom Editores.

Moral, J. (2011). Propiedades psicométricas de un formato de aplicación simplificado para el Inventario de Depresión de Beck. Revista Mexicana de Investigación en Psicología Social y de la Salud, 1(1), 1-15.

Moral, J. (2012). Validación de la escala de pensamiento mágico: profecías apocalípticas y afectividad negativa. Ciencia Ergo Sum, 19(2), 1-10.

Moral, J. \& Tovar, C. A. (2012). Creencias en las profecías del final del mundo en 2012. Estado de ánimo y pensamiento mágico. Psicología desde el Caribe, 29, 330-359. 
Murphy, K. R. \& Myors, B. (2003). Statistical power analysis: A simple and general model for traditional and modern hypothesis tests (2a. Ed.). Hilldale, NJ: Lawrence Erlbaum Associates.

Myers, K. \& Winters, N. (2002). Ten-year review of rating scales. II: Scales for internalizing disorders. Journal of the American Academy of Child and Adolescent Psychiatry, 41, 634-659.

Penley, J. A., Wiebe, J. S. \& Nwosu, A. (2003). Psychometric properties of the Spanish Beck Depression InventoryII in a medical sample. Psychological Assessment, 15, 569-577.

Piccinelli, M. \& Wilkinson, G. (2000). Gender differences in depression. British Journal of Psychiatry, 177, 486-492.

Rocha, S. \& Díaz-Loving, R. (2005). Cultura de género: la brecha ideológica entre hombres y mujeres. Anales de Psicología, 21, 42-49.

Ruscio, A. M. \& Ruscio, J. (2002). The latent structure of analogue depression: Should the Beck Depression Inventory be used to classify groups? Psychological Assessment, 14, 135-145.

Sanz, J., Perdigón, L. A. \& Vázquez, C. (2003). Adaptación española del Inventario para la Depresión de Beck-II (BDI-II): 2. Propiedades psicométricas en población general. Clínica y Salud, 14, 249-280.

Sanz, J., García, M. P., Espinosa, R., Fortún, M. \& Vázquez, C. (2005). Adaptación española del Inventario para la Depresión de Beck-II (BDI-II): 3. Propiedades psicométricas en pacientes con trastornos psicológicos. Clínica y Salud, 16, 121-142.

Sartori, R. (2006). The bell curve in psychological research and practice: myth or reality? Quality and Quantity, 40, 407-418.

Shenal, B. V., Harrison, D. W. \& Demaree, H. A. (2003). The neuropsychology of depression: a literature review and preliminary model. Neuropsychology Review. 13, 33-42.

Spielberger, C. D., Gorsuch, R. L. \& Lushene, R. E. (1970). STAI. Manual for the State-Trait Anxiety Inventory
(Self-evaluation questionnaire). Palo Alto, California: Consulting Psychologists Press.

Spielberger, C. D. \& Díaz-Guerrero, R. (1975). IDARE. Inventario de Ansiedad: Rasgo-Estado. México: Editorial El Manual Moderno.

Sociedad Mexicana de Psicología (2007). Código ético del psicólogo (4a. Ed.). México: Trillas.

Toufexis, D. J., Myers, K. M. \& Davis, M. (2006). The effect of gonadal hormones and gender on anxiety and emotional learning. Hormones and Behavior, 50, 539-549.

Uliaszek, A. A., Zinbarg, R. E., Mineka, S., Craske, M. G., Sutton, J. M., Griffith, J. W., Rose, R., Waters, A. \& Hammen, C. (2010). The role of neuroticism and extraversion in the stress-anxiety and stress-depression relationships. Anxiety, Stress \& Coping, 23, 363-381.

Vázquez, C. \& Sanz, J. (1997). Fiabilidad y valores normativos de la versión española del inventario para la depresión de Beck de 1978. Clínica y Salud, 8, 403-422.

Watson, D. (2000). Mood and temperament. NY: Guilford Press.

Watson, D., Clark, L. A. \& Tellegen, A. (1988). Development and validation of brief measures of positive and negative affect: The PANAS Scales. Journal of Personality and Social Psychology, 54, 1063-1070.

Wiebe, J. S. \& Penley, J. A. (2005). A Psychometric Comparison of the Beck Depression Inventory-II in English and Spanish. Psychological Assessment, 17, 481-485.

Woolfolk, R. L. \& Allen, L. A. (2007). Treating somatization. A cognitive-behavioral approach. NY: The Guilford Press.

Yuan, K. H. \& Bentler, P. M. (2004). On chi-square difference and $\mathrm{z}$-tests in mean and covariance structure analysis when the base model is misspecified. Educational and Psychological Measurement, 64, 737-757.

Zung, W. W. (1965). A self rating depression scale. Archives of General Psychiatry, 12, 63-70. 